\title{
ERRATUM
}

\section{Daytime spikes in dopaminergic activity drive rapid mood-cycling in mice}

MM Sidor, SM Spencer, K Dzirasa, PK Parekh, KM Tye, MR Warden, RN Arey, JF Enwright, JPR Jacobsen, S Kumar, EM Remillard, MG Caron, K Deisseroth and CA McClung

Molecular Psychiatry (2015) 20, 1479-1480; doi:10.1038/mp.2015.8; published online 17 February 2015

Correction to: Molecular Psychiatry (2015) 20, 1406-1419; doi:10.1038/mp.2014.167; published online 6 January 2015
As the result of an editing error, the image in Figure 2e was duplicated in Figure 2f. The correct version of Figure 2 appears below. The publisher regrets the error.

Figure 2. Time-specific alterations in VTA dopaminergic activity in Clock 19 mice. (a) Two-way analysis of variance of firing rate found a significant genotype effect $\left(F_{1,112}=12.67, P=0.0006\right)$. Post hoc tests revealed significant differences in the firing rate of dopaminergic neurons during the first $6 \mathrm{~h}$ of the light cycle and the last $6 \mathrm{~h}$ of the dark cycle $(P<0.05$ using Student's $t$-test; $n=14 / 14 / 16 / 12$ and $14 / 14 / 17 / 12$ for the number of dopaminergic neurons analyzed in WT $(n=8)$ and Clock 19 mice $(n=9)$, respectively). (b) Two-way analysis of variance of bursting rate found a significant genotype effect $\left(F_{1,112}=8.6, P=0.004\right)$ with post hoc analyses revealing significant differences in the bursting rate of dopaminergic neurons during the first $6 \mathrm{~h}$ of the dark cycle $(P<0.05$, by Student's $t$-test; $n=14 / 14 / 16 / 12$ and $14 / 14 / 17 / 12$ for the number of dopaminergic neurons analyzed in WT and Clock 19 mice, respectively). (c) Relative abundance of TH mRNA normalized to the expression of Gapdh. Two-way analysis of variance revealed a significant genotype effect $\left(F_{1,45}=9.42, P=0.004\right)$ with a specific increase in TH expression at ZT4 in Clock 19 mice $(P<0.05, n=3-5$ animals per genotype per time point). Diurnal variation was significant in wild-type (WT) mice (CircWave: $\left.F_{2,27}=10.63, P=0.0004\right)$ but not in Clock 19 mutants $(P>0.05)$. (d) A significant main effect of time was found for total TH $\left(F_{3,28}=5.34, P=0.005\right)$, with an increase in Clock $\Delta 19$ mouse TH levels at ZT9 $(P<0.01)$. Diurnal variation was statistically significant in mutants (CircWave: $F_{2,17}=5.15, P=0.02$ ). (e) There was a significant effect of time on phosphorylated TH (ser 40 ) protein $\left(F_{3,32}=6.50, P=0.002\right)$, with Clock 19 mice exhibiting a specific increase in THser40 levels at ZT9 $(P<0.05$, Student's $t$-test). Diurnal variation in THser40 was statistically significant in mutants (Circwave: $F_{2,17}=7.18, P=0.005$ ). (f) No differences in phosphorylated THser31 protein levels were found at any time point measured. Inset depicts average protein levels over $24 \mathrm{~h}$. (e, f) Dopamine synthesis assay. (g) Dopamine synthesis was significantly increased in Clock $\Delta 19$ mutant mice as measured by L-Dopa in the nucleus accumbens after NSD-1015 administration during the light phase, at ZT4 $\left(t_{9}=2.546, P=0.03\right)$. (h) Dopamine synthesis was unaltered in the dorsal striatum of Clock $\Delta 19$ mutants $(P>0.05, n=5-8$ per group; dark phase $=$ ZT16). White and dark bars below graph represent daytime and nighttime measurements, respectively. 
a

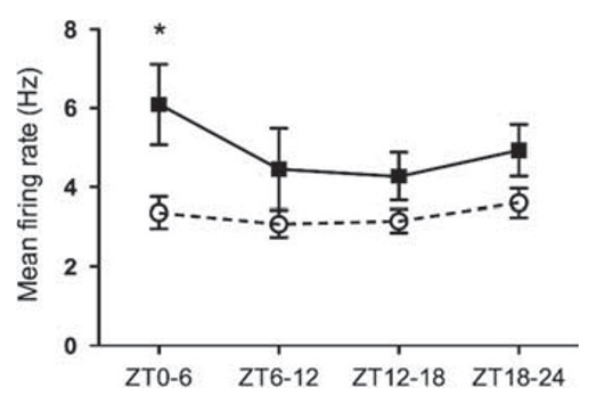

c

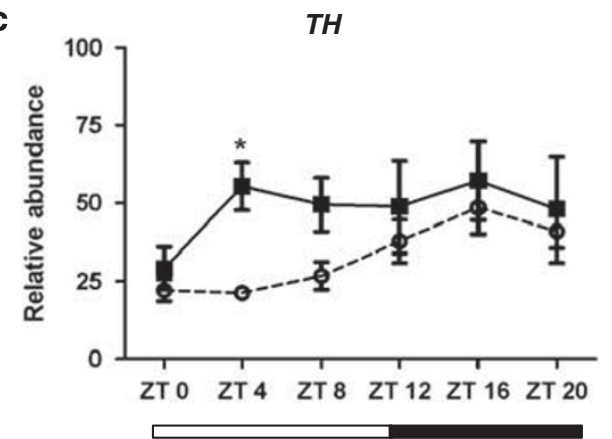

e

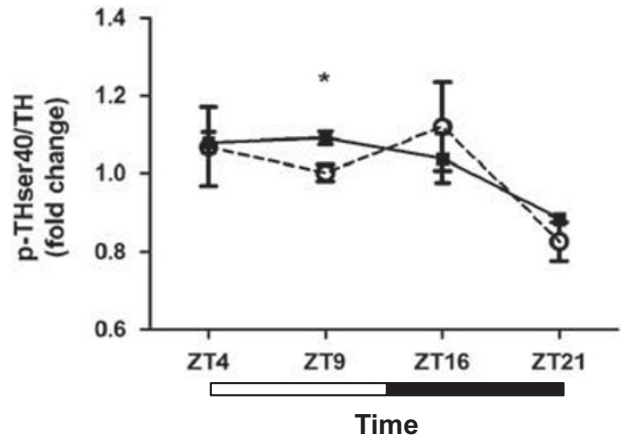

g

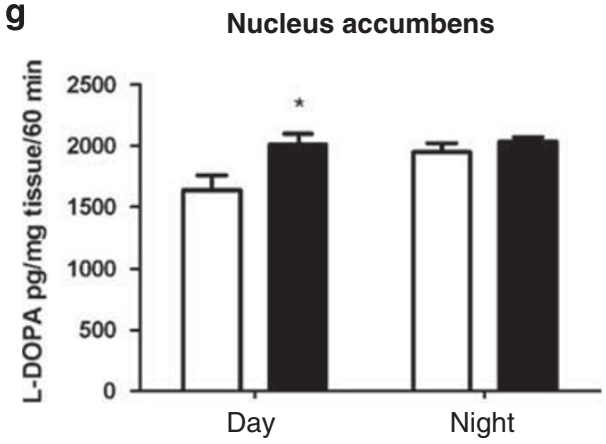

b

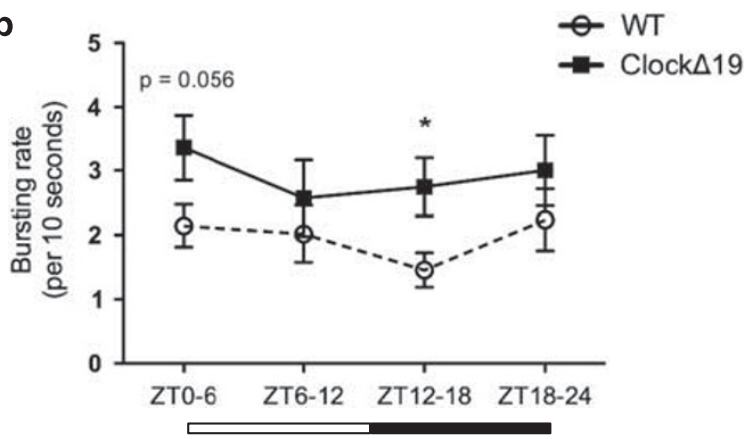

d

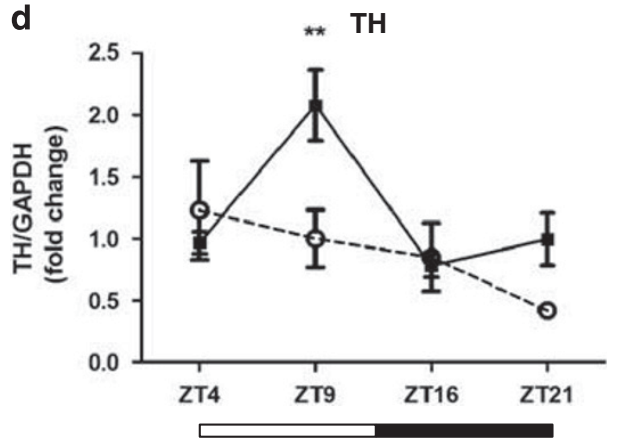

f
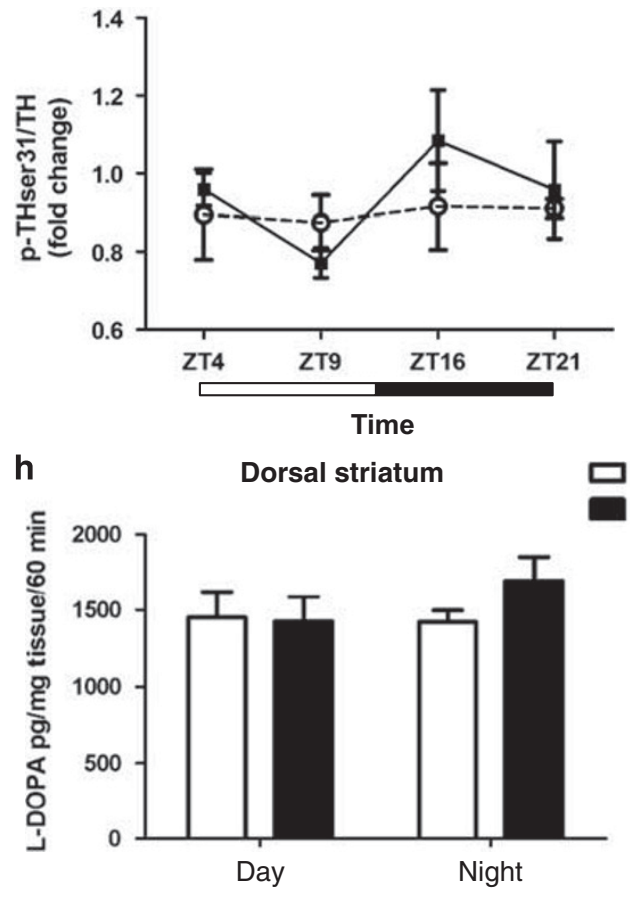